\title{
ATM traffic analysis and control for ABR service provisioning
}

\author{
N.M. Mitrou, K.P. Kontovasilis and E.N. Protonotarios \\ National Technical University of Athens \\ Computer Science Division, \\ Heroon Polytechneiou 9, Zografou \\ GR 157-73, Athens, GREECE \\ Phone: +30-1-7721639, Fax: +30-1-7757501 \\ e-mail: mitrou@phgasos.ntua.gr
}

\begin{abstract}
In this paper the main traffic analysis and control problems related with the ABR service are addressed, modelled and answered on the basis of effective rates defined for the multiplexed connections. Emphasis is given to an adaptive shaping function as a response to congestion feedback. A simple example demonstrates the proposed shaping scheme.
\end{abstract}

\section{Keywords}

ATM, ABR service, fluid-flow modelling, effective rate, adaptive shaping

\section{INTRODUCTION}

The Available-Bit-Rate (ABR) service is being designed as a low-cost transport service over ATM, which will be using those network resources (link bandwidth, in particular) that are left available by other high-priority connections (belonging to the Guaranteed-QoS or G-QoS class). The penalty paid is the lack of any QoS guarantee in terms of transport delay figures, but this may be perfectly affordable by many applications, like file transfer, LAN interconnection, even by some real-time video applications (e.g. MPEG-coded video stream transfer).

Although the idea sounds quite simple, there are several difficulties related not only to the implementation of an ABR service, but also to the analysis and control of the carried traffic. Apart from a priority mechanism for serving the two classes, large buffers are required at each multiplexing/switching stage to store the ABR traffic, in order to maintain the stringent cellloss requirements that are usually imposed by the ABR-serviced applications. A feedback 
mechanism is also necessary to inform preceding nodes/terminals about the congestion conditions along upstream nodes. Additional enhancements are further necessary at the terminals to be able to adapt their traffic profile to different network congestion conditions.

The purpose of this paper is to cope with some of the basic traffic engineering issues related to an ABR service implemented on ATM. Bandwidth allocation, Connection Admission Control (CAC) and traffic shaping are among these issues (CCITT, 1992). Our approach is based on the fluid-flow modelling of the multiplexed traffic (Anick 1982, Baiocchi 1992, Kontovasilis 1994, Kontovasilis 1995, Kosten 1984, Mitra 1988, Mitrou Stern 1991). The large buffer size and the very small overflow probabilities required by an ABR service fully justify this choice. Simple queuing arguments and basic fluid-flow-analysis results lead to the assessment of the performance of an ABR multiplexer, which is further utilised to handle the main traffic control problems outlined above.

A central point in the traffic analysis and control methods considered in this paper is the calculation of an effective or equivalent rate for each of the multiplexed ABR-class streams, in terms of the main traffic and QoS parameters (Elwalid 1993, Guerin 1991, Kelly 1991, Kesidis 1993, Mitrou - St. Peters. 1995). In the case of ON/OFF Markovian streams, a closedform expression greatly simplifies most of the traffic control problems (Mitrou, St. Peters. 1995). Traffic shaping, in particular, is tackled in the above context through changing the source profile (e.g. the peak or the mean rate) according to the loading/congestion conditions prevailing along the connection's path. The calculation of the appropriate profiles is based on the inversion of the effective-rate formula.

Handling the statistical multiplexing problem on the basis of effective rates, implicitly solves one of the major issues arising in an ABR service environment: that of the fair sharing of the available bandwidth among the multiplexed connections and, even further, charging each user accordingly.

In section 2, following this introduction, the implementation and modelling assumptions concerned with the ABR service are presented and some basic results from the related traffic theory are reviewed. The latter are drawn either from the general queuing theory or as more specific derivations of the fluid-flow method. Section 3 addresses the main traffic control problems related with an ABR service, and proposes solutions to them, under the light of the results of section 2 . In section 4 the solutions to the main control problems (bandwidth allocation, connection admission control and shaping) proposed in section 3 are implemented in terms of approximate analytical formulas, relating the effective rate with the other traffic, system and QoS parameters. In the same section a simple example is presented which illustrates a proposed adaptive shaping scheme. Finally, section 5 draws some concluding remarks.

\section{MODELLING ASSUMPTIONS AND SYNOPSIS OF RELEVANT QUEUING RESULTS}

Figure 1 shows a single multiplexing stage with two classes sharing the same link capacity, $C$. One class is of the guaranteed-QoS type having an absolute time priority on the link, i.e. cells of this class are always served first, if present in the queue. The second class is of the ABR type, i.e. it is only served if no cells from the other class are waiting for transmission. Either a 
common buffer or separate buffers may be used for the two classes. In the sequel a common buffer configuration is assumed. In the case, however, of implementing the ABR service by an add-on equipment to existing single-class switches, a separate buffer is obviously necessary.

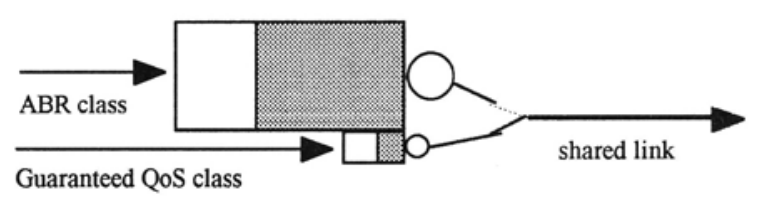

Figure 1 ABR service supporting multiplexer

We now list some assertions that will be of help later on:

\section{Assertion 1}

The total buffer occupancy distribution does not depend on the time priority scheme used, as far as the server keeps on working in the presence of cells waiting for transmission (no matter from which class).

Although rather obvious, the above statement is very useful in many situations, since changing the priority scheme may give rise to easier models to solve. Specific applications of it will be exposed later.

\section{Assertion 2 (Finite- versus infinite-buffer modelling)}

The buffer occupancy complementary probability distribution (CPDF) of an infinite-buffer configuration is always an upper bound to the respective finite-buffer one, thus providing a conservative estimate of the overflow probability or delay percentiles in the latter. The larger the buffer and/or the smaller the overflow probability enforced on the finite boundary, the tighter the bound becomes.

Assertion 2 stems from the fact that in an infinite-buffer system information is always stored and never rejected, thus the probability of exceeding a certain buffer threshold is higher than in the finite variant. The infinite-buffer boundary conditions are always much easier to solve and give a solution independent of the buffer dimension. Furthermore, the ATM systems are usually engineered for small overflow probabilities, in which case the infinite-buffer solution is quite satisfactory in practice.

\section{Assertion 3 (Buffer occupancy seen by arriving cells)}

The buffer occupancy CPDF seen at instances of cell arrivals $H(x)$ and the global CPDF at a random time instance $G(x)$, are related by the simple relationship

$H(x)=\frac{G(x)}{\rho}$,

where $\rho$ is the average load, normalised over the output link capacity. 
This relationship, which is a kind of Little's formula for distributions, has been proven for Markovian fluid models in (Kontovasilis, Annals 1994). However its validity is conjectured to be more general (because it involves only the occupancy distributions and the mean load).

\section{Assertion 4 (Buffer occupancy seen by different classes in heterogeneous mixes)}

In a heterogeneous traffic multiplexing environment, arriving cells of different classes observe, in general, a different CPDF at the buffer. If (1) holds true, then the following relationship does also hold

$$
G(x)=\sum_{i} \rho_{i} H_{i}(x)
$$

where $\rho_{i}$ is the normalised load of and $H_{i}(x)$ the occupancy CPDF seen by class $i$.

The proof for Markovian fluid models is given in (Kontovasilis, Annals 1994), as for the previous assertion.

\section{Assertion 5 (Mixing of Constant Bit Rate (CBR) and non-CBR traffic)}

A CBR component sees always the global CPDF, $G(x)$, while the remaining traffic (provided that (1) holds true) sees a CPDF equal to

$$
H_{\text {non- } C B R}(x)=\frac{1-\rho_{C B R}}{\rho_{\text {non- } C B R}} G(x) \text {. }
$$

\section{Proof}

Due to Assertion 1 above, the buffer occupancy CPDF does not depend on the time priorities assigned to the different classes of the multiplexed traffic. If we assign a high priority to the CBR traffic, then this is equivalent to a system loaded by only non-CBR traffic and featuring an output link of $C\left(1-\rho_{C B R}\right)$. Thus at an instance of (now non-CBR only) cell arrival we have, using (1),

$$
H(x)=\frac{G(x)}{\frac{C \rho_{\text {non }-C B R}}{C\left(1-\rho_{C B R}\right)}}=\frac{1-\rho_{C B R}}{\rho_{\text {non }-C B R}} G(x) .
$$

Now, returning to the original setting, $H(x)$ as just computed is $H_{n o n-C B R}(x)$ and from (2):

$H_{C B R}(x)=\frac{G(x)-H_{n o n-C B R} \rho_{n o n-C B R}}{\rho_{C B R}}=G(x)$, which completes the proof.

We now state two assumptions upon which further developments are based:

\section{Assumption 1:}

The delay requirements of the G-QoS class impose a small buffer occupancy for that class. 
This will be assured by the Connection Admission Control functions, through keeping the load of the G-QoS class low enough to maintain the affordable delay percentiles. The simplest treatment of this issue consists in allocating peak rates to G-QoS class connections. In that case only a small buffer is required, just to absorb the cell-level congestion stemming from the simultaneous cell arrivals.

\section{Assumption 2}

A large buffer is provided for the ABR class to avoid cell losses or to keep the probability of such losses acceptably low.

From Assumption 1 above, the buffer occupancy distribution for the ABR class is approximately equal to the total occupancy distribution and, by virtue of Assertion 1, it can be calculated by applying any analysis method for the aggregate traffic (from both classes), with or without priorities. The fluid-flow method with an infinite buffer, in particular, is ideally suited for this case, due to the large dimension of the buffer and the very low target overflow probability.

\section{TRAFFIC CONTROL FOR AN ABR SERVICE}

This section addresses the main traffic control problems related with an ABR service and proposes some solutions to them, under the light of the discussion in section 2. Connection Admission Control (CAC) is first considered for the two classes (G-QoS and ABR), in the absence of any traffic-profile-adaptation mechanism at the terminals. Then, we assume that the latter have the ability to change (shape) their traffic profile according to the congestion conditions prevalent in the network nodes. The related function is called Traffic Shaping and is examined in sub-section 3.2. In this case CAC is greatly simplified for both classes.

\subsection{Bandwidth Allocation and Connection Admission Control}

The role of the CAC is to ensure that the acceptance of a new connection through a series of multiplexing/switching stages (nodes), as the one depicted by Figure 1, will not violate the QoS contract for the already-established connections through the same nodes. This is equivalent to answering a bandwidth allocation problem at each multiplexing stage, formulated as follows: Given a number of connections and a QoS figure, calculate the bandwidth required to serve this group of connections. We assume the following guaranties for each connection of the two classes:

i) for the G-QoS class
(a) a certain delay percentile: $\operatorname{Pr}\{$ delay $>D\}<\varepsilon_{D}$
(b) a certain maximum cell-loss probability"

\footnotetext{
* When a common buffer with the ABR class is used, a common cell-loss criterion is set for both classes and guarantying (iia) usually suffices. If a separate buffer is devoted to the G-QoS class, usually small, then a separate cell-loss criterion becomes necessary also for this class.
} 
ii) for the $A B R$ class

(a) a certain maximum cell-loss probability

(b) a minimum throughput (mean rate).

Based on the discussion of section 2, the following acceptance conditions must be checked:

I) for a new $G-Q o S$ connection

1. the already established G-QoS connections and the new one should form a set that respects (ia) when served by the total output capacity $C$.

2. the new connection and the already established ones from both classes should form a set that respects (iia) and (iib) when served by the total output capacity $C$.

II) for a new $A B R$ connection

1. the new connection and the already established ones from both classes should form a set that respects (iia) and (iib) when served by the total output capacity $C$.

A very simple peak-rate-based acceptance condition can be adopted for the G-QoS class, in which case the delay for that class comes only from the cell-level congestion (usually quite small, satisfying (ia)). Then, the challenging issue is the satisfaction of the second condition above, aiming at the ABR class guarantee. In the remaining part of this section we confine ourselves to that condition, with the assumption of fixed, non-adaptable traffic profiles for the $\mathrm{ABR}$ connections (i.e. no capabilities for adaptive shaping).

Checking the acceptance condition (I.1 or II.1) can be based on the multiplexing of two different traffic classes served without priorities and sharing the same buffer. The following approaches to that are applicable (Mitrou, ETT 1994):

\section{A) Logical Partitioning of the output capacity}

It consists of calculating the required capacity for each of the two classes with the requirement of (iia), adding the two capacities and checking whether the sum is less than or equal to $C$. This can be done by using either any analysis method available or approximate formulas.

Obviously this is a conservative approach since no multiplexing between the two classes is considered. In the extreme case where the G-QoS connections are of the CBR type then, indeed, this class does not contribute to the multiplexing gain and the above procedure becomes exact. In this case, equations (1), (3) are directly applicable.

B) Using an heterogeneous-traffic-analysis method (Baiocchi 1992, Kontovasilis Annals 1994, Stern 1991) to assure (iia)

This approach suffers from a large computational overhead that may stress the time-constraints of the Connection Admission procedure. 
C) Allocating peak rates to the G-QoS connections together with shaping of the ABR streams to ensure adaptation to the changing availability of bandwidth

This is a promising approach that matches well to the effective rate schemes; it is discussed further in section 3.2.3.

The CAC (as well as the other traffic control functions considered later) is facilitated by using effective rates to characterise the bandwidth requirements of each connection in the given multiplexing environment, with the specified QoS requirements (see also the next sub-section). In section 4 an approximate closed-form expression relating the Effective Rate of a traffic stream with the basic traffic and QoS parameters is given, which greatly simplifies the CAC procedures outlined above.

\subsection{Traffic shaping for the ABR service}

Traffic shaping aims at producing profiles which exhibit certain parameter values. The simplest traffic shaping operation is the peak-rate enforcement, keeping the peak rate of a stream less than or equal to a pre specified value (Guillemin 1992). Other shaping operations, like mean or maximum burst-size enforcement or burstiness enforcement, are applicable on bursty traffic streams.

Here we examine traffic shaping functions that are necessary to enforce specific effective rate values, i.e. to produce streams with a specific multiplexing attitude. By definition, the ABR service utilises a bandwidth that is not constant, in general, but varies according to the aggregate rate fluctuations of the G-QoS connections. Moreover, the ABR connections themselves are bursty in nature and have variable rate requirements. It becomes therefore obvious that the notion of an effective (or equivalent) bandwidth is ideally suited for this case.

\subsubsection{Effective Rate enforcement}

There exist various terms (effective rate, effective bandwidth; equivalent bandwidth etc.) in the literature, to address the notion of a descriptor for the bandwidth requirements of a bursty traffic stream for a certain buffer overflow probability or a delay percentile (Elwalid 1993, Guerin 1991, Kesidis 1993, Mitrou - ETT 1994). Some of the definitions are concerned with each stream in isolation (Elwalid 1993, Guerin 1991, Kesidis 1993), in which case any gain comes only from buffering, while other consider them in a multiplexing environment (Mitrou ETT 1994), where an additional gain comes from multiplexing. Depending on the buffer size (compared to the burst size) and the burstiness of the multiplexed streams one or the other of the two parts may prevail.

Here we consider the more general definition of the effective rate (Mitrou - ETT 1994):

Given that $N$ traffic streams of a certain fluid class are multiplexed together, there can be found a unique output rate $C_{N}$, such that $\operatorname{Pr}\left\{\right.$ queue $\left.>V_{b}\right\}=p$ (specified). Then $R_{e}=C_{N} / N$ is defined as the effective rate (or effective bandwidth), required by each stream within this multiplexing environment. 
Calculating the $R_{e}$ as a function of $N$ and making a suitable interpolation between the discrete points $\left(C_{N}, C_{N} / N\right), N=2,3, \ldots$ (a linear interpolation is sufficient in the context of this work), a continuous curve is derived allowing the definition of $R_{e}$ for a fixed output rate (instead of fixing the number of multiplexed streams). The continuous alternative permits, in principle, an extension to cases where heterogeneous streams share a common output link.

To simplify things, we further assume streams of the ON/OFF type. This assumption is not unrealistic in an ABR service environment, where the traffic sources either transmit at a constant rate or remain silent, as a result of the shaping operation. In that case the effective rate is a function of the form

$R_{e}=f\left([V, r, c],\left[V_{b}, C\right], p\right)$,

where $[V, r, c]$ is the traffic descriptor of the stream (in terms of its mean rate, $r$, the peak rate, $c$, and the mean burst size, $V),\left[V_{b}, C\right]$ is the multiplexer's dimensions (in terms of the buffer size, $V_{b}$, and the output link rate, $C$ ) and $p$ is the QoS requirement. A traffic descriptor equivalent to $[V, r, c]$ above is the $[V, B, c]$, where $B \equiv c / r$ is the burstiness of the stream.

As far as the shaping function is concerned with, each of the three traffic parameters given above can be controlled so as to keep the effective rate of the stream constant. This way, different shaping schemes can be devised.

Peak-rate shaping for effective rate enforcement

Equation (4) is solved for $c$ to yield

$c=f_{1}\left(R_{e}, V, r, V_{b}, C, p\right)$.

By fixing the independent parameters in (5) we can determine and enforce a specific value for the peak rate.

Mean-rate shaping for effective rate enforcement

We can instead solve (4) with respect to the mean rate, $r$

$r=f_{2}\left(R_{e}, V, c, V_{b}, C, p\right)$

The mean rate, calculated by (6), can be regulated by controlling the silence intervals between successive bursts, while the peak rate is kept constant. It must be noticed that, while $c$ is a deterministic parameter, $r$ is a statistical average. If, for the purpose of shaping, we enforce a silence between bursts that is deterministically calculated by (6), the assumptions under which (4) has been derived may be violated. A usual assumption, for example, is for exponentially distributed bursts and silences. It has been found, however, that deterministic values for the $V$ and/or the $r$ (or, more generally, hypo-exponentially distributed values) lead to a better performance than their exponential or hyper-exponential counterparts (Mitrou - Bradford 
1994). Thus, as far as the multiplexing behaviour of the shaped streams is concerned, we are on the safe side by enforcing constant silence values.

For both shaping schemes described above the principal problem focuses on deriving a formula like (4) and inverting it to get (5) or (6). In the next section approximate formulas of the type of (4) will be presented, which provide a solution to that problem.

\subsubsection{Adaptive shaping}

When the traffic, system and QoS parameters involved in equations (4) through (6) remain constant or stationary, the values calculated for the controlled parameters $(c$ or $r)$ are kept constant for the life-time of a connection. This is referred to as fixed shaping.

The above case, however, of constant or stationary parameters, is only the exception. Hardly one finds a source that can guarantee the stationarity of the volume of the produced bursts, for example. Also the number of the multiplexed connections changes dynamically. It is therefore necessary to apply an adaptive shaping, which will take into account the short-term parameter values of the produced traffic as well as the loading conditions at the multiplexers and change the traffic profile accordingly.

The application of an adaptive shaping in ABR service provisioning is shown in fig. 2 . According to this figure, each source of the ABR type gets a feedback from the network about the loading and congestion conditions prevailing at ABR switches. This information may be generated periodically or after a significant change has occurred (e.g. the number of established connections has changed significantly or the content of the ABR buffer has exceeded some threshold). For a logical partitioning of the bandwidth between G-QoS and ABR class, as discussed in 3.1, the information that has to be fedback to the shaper is the available for the ABR class capacity $C_{a v}$, the available at that time buffer capacity $V_{a v}$, the target overflow probability $p$, and the effective rate allocated to the source. The shaper utilises this information, along with the one extracted by monitoring its own output traffic, to change the regulated parameter levels accordingly.

\subsubsection{CAC combined with adaptive shaping}

If the terminals are equipped with an adaptive shaper, the CAC algorithm may be significantly simplified, providing for e.g. peak-rate allocation to the G-QoS connections and effectiverate allocation to the ABR class. It is up to the shaping mechanism to adapt to the loading and congestion conditions, in order to enforce the contracted effective rates.

Since peak rate is allocated to the G-QoS class, admission of new and release of terminated G-QoS connections can be modelled by introducing a discrete finite set of states $J=1, . ., K$, each associated with a rate-value $C_{J}$, expressing the collective bandwidth requirements of the G-QoS class in that particular state. Without essential restriction on generality, one may assume Markovian transitions between these states, since arbitrary sojourn times may be approximated by Markovian modelling - at the expense of increasing the statespace.

The adaptive shaping consists of tuning one or more parameters of each ABR stream (with possibly different controlling parameters among $\mathrm{ABR}$ streams of differing natures) so as 
to adjust to the fluctuations of the bandwidth consumed by the G-QoS class. It is assumed that, while the parameter values may be freely tuned, the number of states describing each $A B R$ stream remains constant; this is not restricting for all the controls discussed in this paper. It is further assumed that the dynamics governing G-QoS state-changes are much slower, as compared to the burst-dynamics of the ABR connections; this is a fairly natural assumption, given that the G-QoS state-changes correspond to call-level phenomena. Under this assumption, the global model for the compound traffic belongs to the class of Nearly Completely Decomposable Markovian Fluid Models.

By employing results from the relevant theory (Kontovasilis 1995) it can be shown (Mitrou - Telecom. Systems 1995) that any adaptive control conforming to the description above is stable, in the following sense: given that, for all $J$, the tuning of the controlling parameter(s) results in satisfying the QoS criterion within a particular G-QoS state $J$ (i.e., by assuming that the G-QoS bandwidth requirements remain time-invariant and equal to $C_{J}$ ) then the quality criterion is respected globally, viz. with G-QoS rate fluctuations present.

This result is independent from the from of control applied, given that a simple loading condition applies, namely that for each state $J$, the sum of the compound G-QoS rate $C_{J}$ plus the aggregate mean rate of all $\mathrm{ABR}$ streams, as controlled within state $J$, does not exceed the link capacity. This loading condition is always satisfied when enforcing the connection acceptance conditions in the beginning of section 3.1. In particular, the loading condition is satisfied when the control is based on an effective rate notion. For more details and a proof on this stability issue, see (Mitrou - Telecom. Systems 1995).

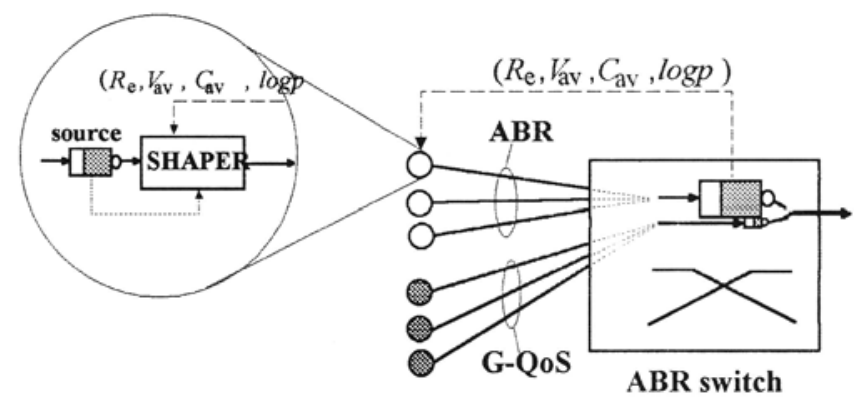

Figure 2 Adaptive shaping of the ABR-serviced traffic

\section{APPROXIMATE CLOSED-FORM CALCULATION OF THE TRAFFIC CONTROL}

In (Mitrou - St. Peters. 1995) an approximate calculation of the effective rate of ON/OFF Markovian streams is presented in closed form. The formula is derived by fitting experimental data for large buffer sizes and burstiness values (such conditions fit quite well into an ABR service environment). 
In particular, by optimising the fitting in the least-squares sense over the range

$20<\frac{V_{b}}{V}<200, \quad 5<B<100, \quad-3<\log (p)<-9$

we get:

$\frac{c-R_{e}}{R_{e}-r} \cong a \sqrt{\frac{C}{c}}+b$

$a \cong\left[0.075+e^{0.4 \log p-0.7186}+e^{-0.03 \frac{V_{b}}{V}-1.3}\right] B+0.3$,

$b \cong\left[\left(0.035+e^{0.3321 \log p-1.28}\right) \frac{V_{b}}{V}-1.1\right] B+\left(0.043+e^{0.281 \log p-1.247}\right) \frac{V_{b}}{V}-1.7$

where the $\log$ is in base 10 .

A cruder approximation is derived by using the asymptotic (dominant-eigenvalue) behaviour of the buffer-content CPDF (Anick 1982). This results in a conservative approximation

$\ln p \leq-\frac{c}{c-r} \frac{1-r N / C}{1-C / c N} \frac{V_{b}}{V}=-\left(\frac{c}{c-r}\right) \frac{1-r / R_{e}}{1-R_{e} / c}\left(\frac{V_{b}}{V}\right)$.

In (9) the effective rate $R_{e}$ does not depend on the output capacity, but only on the traffic stream characteristics and the QoS point (buffer size, overflow probability). This is the effective rate as defined in (Elwalid 1993, Guerin 1991), taking into account only the buffering.

For very large peak rate values (i.e. the bursts enter the queue "instantly"), the right-hand side of (9) tends to $-\left(1-r / R_{e}\right) V_{b} / V$, providing a very rough but useful upper bound of the overflow probability.

By using (8) or (9) the traffic control procedures described in the previous section are directly applicable, although not always in closed form.

Currently, we study the possibility of extending the dominant pole approximation to include the dependence of the overflow probability on the output capacity. In this approach the overflow probability is $G(x) \approx \alpha e^{-z_{\omega} x}$, where $z_{\infty}$ is the dominant slope as before (in semilog scale), while $\alpha$ is the Chernoff upper bound to the probability that the cumulative input rate exceeds the link capacity. Study of this approximation for general Markovian fluid models shows that indeed, the dependence of the ER on the link capacity asymptotically occurs in a square-root fashion, as in (8a). 


\subsection{Approximate, analytical Bandwidth Allocation and Connection Admission Control}

Recalling the principle of Logical Partitioning from 3.1, we need to calculate the capacity $X$ that must serve a number $K$ of similar" connections in order to maintain a maximum cell loss probability in a buffer of size $V_{b}$.

By applying (8) for each of the $K$ connections in the group, we have

$\frac{c_{i}-x_{i}}{x_{i}-r_{i}}=a_{i} \sqrt{\frac{X}{c_{i}}}+b_{i}$

with $x_{i}$ denoting the effective rate allocated to the $i_{\text {th }}$ connection and

$\sum_{i=1}^{K} x_{i}=X$

The above equation can be solved with respect to $X$, since we know that the $x_{i}$ 's are continuous (decreasing) functions of $X$. A similar (and simpler) approach can be followed based on (9). For each stream an effective rate is calculated (through solving (9)) and the sum of all these rates gives the required output capacity. If one uses (9) (or its approximation with an infinite rate) instead of (8) then the requirement of similar connections is waived and a heterogeneous environment can be fully accommodated (Elwalid 1993, Guerin 1991).

\subsection{Approximate, analytical calculation of the shaping values}

By substituting in (8b) $B$ with its equal $c / r$ we can solve (8a) with respect to $c$ (a third-order equation) or $r$ (a second-order equation).

Equation (9) is again more manageable. It gives a second-order equation in $c$ and a firstorder one in $r$.

\subsection{An example of rate-based adaptive shaping}

The following example is a simple case of rate-based adaptive shaping. A number $(N=70)$ of identical bursty sources are multiplexed on the same link of a capacity $C$, supported by a buffer of a size $V_{b}=3000$ cells. No connections of the G-QoS class are present (equivalently, we could assume that the considered link capacity $C$ is just what is left for the partition of the ABR class). All the rates will be subsequently normalised with respect to $C$, i.e. $C=1$ will be assumed.

\footnotetext{
* As mentioned before, only similar connections may be grouped together for the above calculations, since the effective rate has been defined in a homogeneous multiplexing environment. Similarity here means not large differences in the mean burst size and the burstiness.
} 
The sources generate data packets of an exponentially distributed size with mean $V=75$ cells, according to a Poisson process such that $r=0.01$. With reference to Figure 2, no monitoring of the produced traffic is necessary by the shapers, since the sources are assumed to be stationary with known characteristics. The sources are informed by the network about the loading \& congestion conditions at the ABR multiplexer. In this simple example the number of connections is assumed fixed $(=70)$ and a feedback is only issued whenever the buffer content crosses a threshold. The rate adaptation mechanism is a two-state machine, as described by the pseudo code of Figure 3. A hysterisis in switching from $c_{2}$ to $c_{1}$ (i.e. $t h r 2<$ thrl) is used to avoid unwanted oscillations around the threshold.

Using (8) we get $c_{1}=0.666$ (it is rounded up to unity, i.e. $c=1$, is used in the uncongested state) and $c_{2}=0.048$. Equation (9) would give more conservative rates, i.e. $c_{1}=0.1817$ and $c_{2}=0.0213$. For the uncongested state, a relatively high overflow probability $\left(p_{1}=10^{-2}\right)$ has been used for the calculation of the respective rate $c_{1}$, in order to increase the

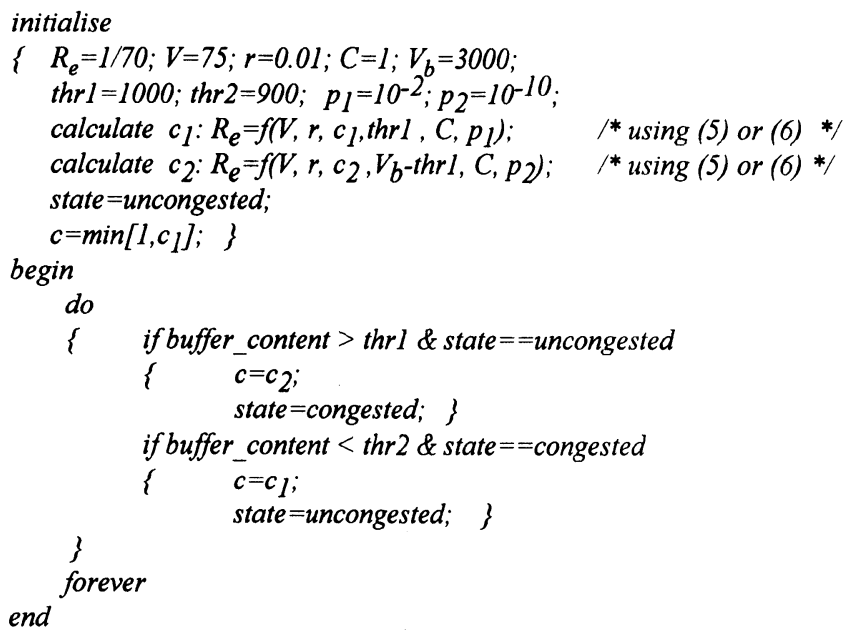

Figure 3 Pseudo code for the example of rate-based adaptive shaping

throughput. This probability does not affect the ultimately offered QoS to the ABR class; it affects only the rate at which feedback messages are issued and, hence, the signalling overhead induced by these messages.

Figure 4 presents the results from a discrete-time simulation of the above example. We can clearly see the two different slopes of the buffer-content probability distribution, corresponding to the uncongested and congested states. A zero delay for the feedback has been used. In a real system, however, there will be a finite response time, which must be taken into account. 


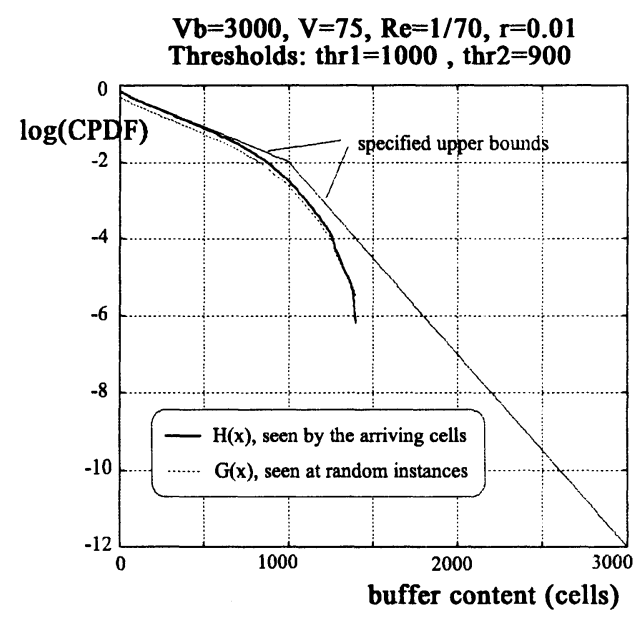

Figure 4 Simulation results of the adaptive shaping example

\section{CONCLUSION}

The main traffic analysis and control problems related with an Available Bit Rate (ABR) service were addressed, modelled and provided with a solution method. The proposed method is based on the notion of Effective Rates defined for the multiplexed connections and calculated approximately, by using a fluid-flow model for the ATM multiplexer. Simple queuing arguments and results from the application of the fluid-flow method on Markovian traffic streams lead to important and analytically simplifying relations between the QoS figures experienced by the two classes, the ABR and the Guaranteed-QoS.

Inverting the Effective Rate equation yields a solution to the specific problems of Bandwidth Allocation, Connection Admission Control and Traffic Shaping in the considered multiplexing environment. An adaptive, rate-based traffic shaping mechanism was proposed and tested through simulation.

\section{REFERENCES}

Anick, D., Mitra, D. and Sondhi, M.M. (1982) "Stochastic theory of a data-handling system with multiple sources", Bell Syst. Tech. J. 61 1871-1894.

Baiocchi, A., Blefari-Melazzi, N., Roverti, A. and Salvatore, F., (1992) "Stochastic Fluid Analysis of an ATM Multiplexer Loaded with Heterogeneous ON-OFF Sources: an Effective Computational Approach," In Proc. INFOCOM' '92, pp. 3C.3.1-3C.3.10.

CCITT STUDY GROUP XVIII (1992) Traffic control and resource management in B-ISDN. CCITT Recomendation I.371, Geneva. 
Elwalid, A. and Mitra, D. (1993) "Effective bandwidth of general Markovian sources and admission control of high speed networks," IEEE/ACM Trans. on Netw. , pp. 329-343.

Guerin, R., Ahmadi, H. and Naghshineh, M. (1991) "Equivalent capacity and its application to bandwidth allocation in high-speed networks," IEEE JSAC, vol. 9, pp. 968-981.

Guillemin, F., Boeyer, P., Dupuis, A. and Romoeuf, L. (1992) "Peak Rate Enforcement in ATM Networks," IEEE INFOCOM '92, paper 6A.1.

Kelly, F.P. (1991) "Effective bandwidths at multi-type queues," Queue. Syst., vol. 9, pp. 5-15.

Kesidis, G., Warland, J. and Chang, C.-S. (1993) "Effective Bandwidths for Multiclass Markov Fluids and Other ATM Sources", IEEE/ACM Trans. on Netw., Vol.1,No.4, pp. 424-428.

Kontovasilis, K.P., and Mitrou, N.M. (1994) "Bursty Traffic Modeling and Efficient Analysis Algorithms via Fluid-Flow Models for ATM IBCN," Annals of Operations Research, Vol. 49, special issue on Methodologies for High Speed Networks pp. 279-323.

Kontovasilis, K.P., and Mitrou, N.M. (1995) "Markov Modulated Traffic with Near Complete Decomposability Characteristics and Associated Fluid Queueing Models," to appear in the Adv. Appl. Prob. Dec. 1995.

Kontovasilis, K.P., and Mitrou, N.M. (1994) "Stochastic fluid models for a buffer loaded by Markov modulated traffic with near complete decomposability characteristics", in IFIP Transactions C-21, "High Speed Networks and their Performance, H.G. Perros and Y. Viniotis eds., North-Holland, pp. 363-397.

Kosten, L., (1984) "Stochastic theory of data-handling systems with groups of multiple sources," in Performance of Computer Communication Systems, H. Rudin and W. Bux, Eds. Amsterdam, The Netherlands: Elsevier, pp. 321-331.

Mitra, D., (1988) "Stochastic theory of a fluid model of producers and consumers coupled by a buffer," Adv. Appl. Prob. 20 pp. 646-676.

Mitrou, N.M., and Koukos, A. (1993) "An effective-rate enforcement algorithm for ATM traffic and its hardware implementation", Proceedings of the IBCN\&S, Copenhagen, April 20-23 1993.

Mitrou, N.M., Kontovasilis, K. and V. Nellas, "Bursty Traffic Modelling and Multiplexing Performance Analysis in ATM Networks: A Three-moment Approach," 2nd IFIP Intern. Workshop on on Performance Modelling and Evaluation of ATM Networks, Bradford, 4-6 June 1994.

Mitrou, N.M., Kontovasilis, K.P., Kroener, H., amd Iversen, V.B. (1994) "Statistical Multiplexing, Bandwidth Allocation Strategies and Connection Admission Control in ATM Networks,"European Transactions on Telecommunications, Vol. 5, No. 2, pp. 161-175.

Mitrou, N.M., Kontovasilis, K.P., and Protonotarios, E.N. (1995) "A closed-form expression for the Effective Rate of ON/OFF traffic streams and its usage in basic traffic control problems", Proceedings of the Intern. Teletraffic Seminar, St. Petersburg, June 1995.

Mitrou, N.M., Kontovasilis, K.P., and Protonotarios, E.N. (1995) "ATM traffic engineering for ABR traffic provisioning", to appear in the Telecommunications Systems Journal.

Stern, T.E., and Elwalid, A.I. (1991) "Analysis of separable Markov-modulated rate models for information-handling systems", Adv. Appl. Prob., vol. 23, pp. 105-139. 